\title{
Video Steganography for Face Recognition with Signcryption for Trusted and Secured Authentication by using PCASA
}

\author{
Kavitha Raju, \\ Ph.D Scholar, \\ St. Peter's University, \\ Avadi, Chennai, \\ Tamilnadu, India
}

\author{
S.K.Srivatsa, \\ Senior Professor, \\ St. Joseph's College of Engineering, \\ Old Mahabalipuram Road, \\ Chennai, India
}

\begin{abstract}
Face recognition systems are very useful in many applications such as surveillance, biometrics and security. Principal Component Analysis (PCA) has also been used in some important applications, especially in pattern detection such as face detection and recognition. In real-time applications, response time must be as fast as possible. For this purpose, we propose a new PCA signcryption implementation for secure authentication by face detection based on the cross-correlation in the frequency domain between the input image and eigenvectors (weights). Simulation results demonstrated that our proposal is faster than the existingmethods. The experimental results for different images also show good performance. In this paper we proposed a face recognition system using the Principal Component Analysis Signcryption Algorithm (PCASA) within video steganography.
\end{abstract}

\section{General Terms}

Face recognition, biometrics, video

\section{Keywords}

PCA, PCASA, Signcryption, Steganography, eigenvectors

\section{INTRODUCTION}

For the past eras, many face recognition systems have been proposed based on principal components analysis (PCA). Even though the particularsare different, all these systems can be described in terms of the same preprocessing and run-time steps. During preprocessing, they register a gallery of $\boldsymbol{m}$ trained images to each other and unroll each image into a vector of $\boldsymbol{n}$ pixel values. After that, the mean image for the gallery is subtracted from each and the resulting images are placed in a gallery matrix M. Element $[i ; j]$ of $\mathrm{M}$ is the $i$ th pixel from the $j$ th image.

A covariance matrix $\Omega=$ MMT characterizes the distribution of the $\boldsymbol{m}$ images in Rn. Subsets of the Eigenvectors of $\Omega$ are used as the basis vectors for a subspace in which to compare gallery and novel probe images. When sorted by decreasing Eigen value, the full set of unit length Eigenvectors represents an ortho normal basis where the first direction corresponds to the direction of maximum variance in the images, the second the next largest variance, etc. These basis vectors are the Principle Components of the gallery images. Once the Eigen space is computed, the centered gallery images are projected into this subspace. At run-time, the recognition is accomplished by projecting a centered probe image into the subspace and the nearest gallery image to the probe image is selected as its match. Here we added signcryption within that probed images for security. Fig. 1 illustrates the steps.
In some systems the images are registered prior to face recognition. Many types of techniques are used to recognize facial features and register them to each other. Different systems may use different distance measures when matching probe images to the nearest gallery image. Different systems select different numbers of Eigenvectors (usually those corresponding to the largest $\boldsymbol{k}$ Eigenvalues) in order to compress the data and to improve accuracy by eliminating Eigenvectors. For helping the people to compare and evaluate, Moon and Phillips created the FERET face database with individual steps of for face recognition process. They alsodid initial comparisons of some common distance measures. This paper extends their work, by using PCASA.

\section{LITERATURE SURVEY}

Jonathon Phillips etalexplained,face recognition is a $\mathrm{K}$ class problem, where $\mathrm{K}$ is the number of known individuals and Support Vector Machines (SVMs) are a binary classification method. The face recognition problem is formulated as a problem in difference space, which models dissimilarities between two faces.

\section{OVERVIEW}

\subsection{Disadvantages of Existing System}

- All previous hardware implementations assume that the input to the face recognition system is an unknown face image without any detail of the image owner.

- Computing complexity.

- Existing system processed the static faces which have the same face expression.

- The existing system cannot tolerate variations in the new face image. It requires the new image to be almost exactly matching with one of the images in the database. Otherwise the resultwill be denial of access for the individual.

- The performance level of the existing system is not appreciable.

\subsection{Advantages of Proposed System}

The principal component analysis (PCA) is one of the most successful techniques and has been used to recognize faces in images. However, high computational cost and dimensionality is a major problem of this technique. Given as-dimensional vector representation of each face in a training group of images, Principal Component Analysis (PCA) tends to find a $\boldsymbol{t}$-dimensional subspace whose basis vectors correspond to the maximum variance direction in the original image space. This 
new subspace is normally lower dimensional $(t<<s)$. If the image elements are considered as random variables, the PCA basis vectors are defined as eigenvectors of the scatter matrix.

\subsection{Advantages}

- Computational cost less because smaller images (main features) require less processing to train the PCA.

- Better recognition accuracy and discriminatory power because using of dominant features and hence can be used as an effective means of authentication.

- Signcryption included for additional security measures.

- Prevents insecure mailing against hacking mechanism.

\section{SYSTEM ARCHITECTURE DESIGN}

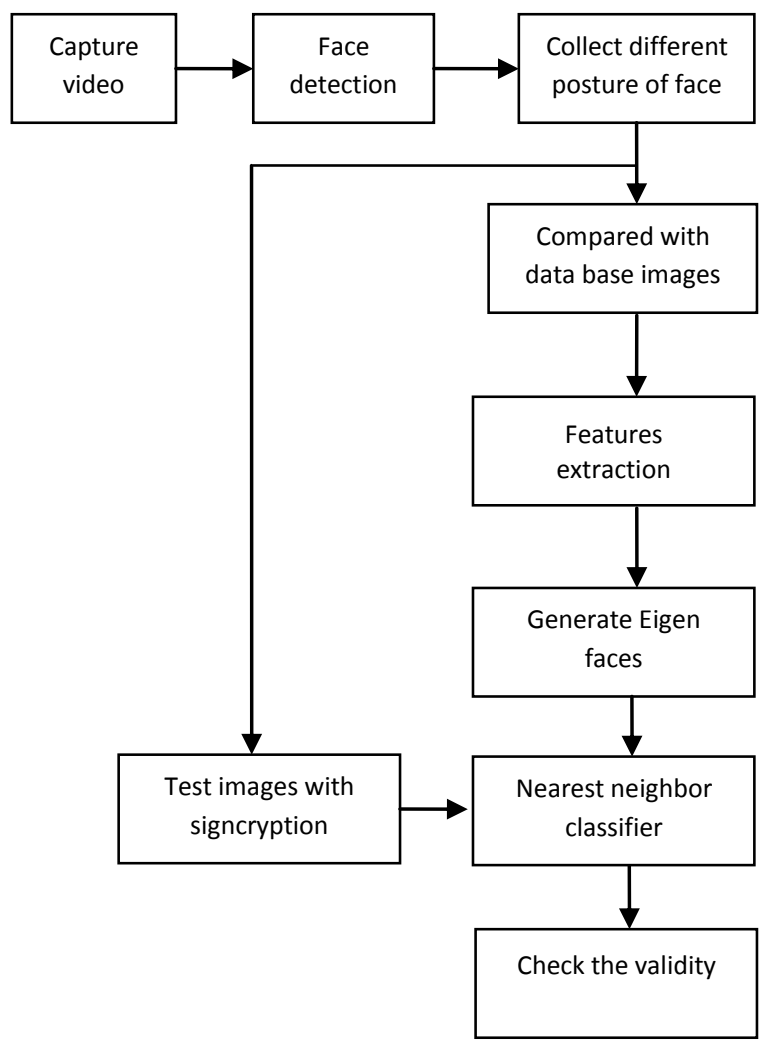

Fig: 1. System Architecture

\section{FEATURE EXTRACTION}

Let there be $\mathrm{R}$ face images in the training set, where each image $\mathrm{Xi}$ is a two dimensional array of size $\boldsymbol{m} \times \boldsymbol{n}$ of intensity values. The image $\mathrm{Xi}$ can be converted into a vector of $\mathrm{D}$ (where $\mathrm{D}=\boldsymbol{m} \times \boldsymbol{n}$ ) pixels. The rows of pixels of the image are placed one after another to form the vector.

If the training set of $\mathrm{R}$ images is defined by $\mathrm{X}=(\mathrm{X} 1, \mathrm{X} 2, \mathrm{X} 3$ ... XR), then the covariance matrix is defined as:

$$
\begin{gathered}
\Gamma=\frac{1}{R} \sum_{I-1}^{R}\left(X_{i}-\bar{X}\right)\left(X_{i}-\bar{X}\right)^{T} \\
=\Phi \Phi^{\mathrm{T}}
\end{gathered}
$$

Where $\Phi=\left(\Phi_{1}, \Phi 2, \Phi 3---\Phi_{\mathrm{R}}\right) \subset \mathfrak{R}^{D \times R}$ and

$$
\bar{X}=\frac{1}{R} \sum_{i-1}^{R} x_{i}
$$

is the mean image of the training set. Also the dimension of the covariance matrix is $D \times D$.

The eigenvalues and eigenvectors are then calculated from the covariance matrix.

Let $Q=\left(Q_{1}, Q_{2},---Q_{R}\right) \subset \mathfrak{R}^{D \times R}($ Generally, $\boldsymbol{r}<R)$ be the $\boldsymbol{r}$ normalized eigenvectors corresponding to rlargest eigenvalues. Each of the $r$ eigenvectors is called an Eigenface. Now, each of the face images of the training set $X$ is projected into the Eigenface space to obtain its corresponding Eigenface based feature $\mathrm{Z}_{\mathrm{i}} \subset \mathfrak{R}^{D \times R}$ which is defined as:

$\mathrm{Z}_{\mathrm{i}}=\mathrm{QTY}_{\mathrm{i}, \quad} \quad \mathrm{i}=1,2 \ldots \mathrm{R}$

Where $\mathrm{Y}_{\mathrm{i}}$ is the mean-subtracted image of $\mathrm{Xi}$.

\section{CLASSIFICATION}

\subsection{Introduction}

The Principal Component Analysis (PCA) is one of the most powerful techniques that have been used in image recognition or face compression. PCA is a statistical method under the broad title of factor analysis. The function of PCA is to reduce the large size of the data space (variables) to the smaller intrinsic dimensionality or size of feature space (independent variables), that are needed to describe the data cost efficiently. This is the case when there is a strong correlation between observed variables.

Since PCA is a classical technique which can perform functions in the linear domain, thus the applications having linear models is much suitable.

The field of face recognition is very useful inmany applications such as security, biometric systems, banks and more. Face recognition can be partitioned into Face identification, Face classification, sex determination; people surveillance in crowded areas, video content indexing, and Personal identification used for purposes like Driver's License etc., Mug shots matching and Entrance security.

The main idea of using PCA for face recognition is to express the large 1-D vector of pixels constructed from the 2-D facial image into the compact principal components of the feature space. This can be called as projection of eigenspace.

Eigen space is calculated by identifying the eigenvectors of the covariance matrix derived from a set of facial images (vectors). Once the eigenfaces have been computed, several types of decision can be made depending on the application. Face recognition is a broad term which is categorized as identification where the labels of individuals must be obtained, categorization where the face must be assigned to a certain class. Recognition of a person, where it must be decided if the individual has already been seen, PCA computes the basis of a space which is represented by its training vectors. These basis vectors, actually eigenvectors, computed by PCA are in the direction of the largest variance of the training vectors called eigenfaces. Each eigenface can be viewed a feature. When a particular face is projected onto the face space, its vector into the face space describes the importance of each of those features in the face. The face is expressed in the face space by its eigenface coefficients. We can handle a large input vector, facial image, only by taking its small weight vector in the face space. This means that we can reconstruct the original face with some error, since the dimensionality of the image space is much larger than that of face space. 
Each face in the training set is transformed into the face space and its components are stored in memory. The face space has to be populated with these known faces. An input face is given to the system, and then it is projected onto the face space. The system computes its distance from all the stored faces.

\subsection{PCASA Implementation}

Principal component analysis with signcryption Algorithm (PCASA) has been proposedto improve the security and maintain the originality of the user image.Signcryption is the method to generate unique image of the user. This is done by gettingmulti gesture video clipsof the user and preprocessed byPCASA

\subsection{PCA Implementation}

Researchers derive algebraic solution to PCA using linear algebra. This solution is based on an important property of eigenvector decomposition. The data set is $X$ which is an $m \times$ $n$ matrix, where $m$ is the number of measurement types and $n$ is the number of samples. The goal is summarized as follows:

Find some orthonormal matrix $P$ where $Y=P X$ such that: $C Y \equiv \frac{1}{n-1} \times Y Y T$ isdiagonalized. The rows of $\mathrm{P}$ are the principal components of $X$. We begin by rewriting $C Y$ in terms of our variable of choice $P$.

$$
\begin{aligned}
& C Y \equiv \frac{1}{n-1} \times Y Y T \\
& C Y \equiv \frac{1}{n-1} \times(P X)(P X) T \\
& C Y \equiv \frac{1}{n-1} \times \mathrm{PXXTPT} \\
& C Y \equiv \frac{1}{n-1} \times \mathrm{P}(\mathrm{XXT}) \mathrm{PT} \\
& C Y \equiv \frac{1}{n-1} \times \mathrm{PAPT}
\end{aligned}
$$

Note that, we defined a new matrix $A \equiv X X T$, where $A$ is symmetric. The roadmap is to recognize that a symmetric matrix (A) is diagonalized by an orthogonal matrix of its eigenvectors. For a symmetric matrix:

$$
A \equiv E D E^{T}
$$

Where, $D$ is a diagonal matrix, $E$ is a matrix of eigenvectors of $A$ arranged as columns. The matrix $A$ has $r \leq \boldsymbol{m}$ ortho normal eigenvectors where $\boldsymbol{r}$ is the rank of the matrix. The rank of $A$ is less than $m$ when $A$ is degenerate or all data occupy a subspace of dimension $r \leq m$ which maintaining the constraint of orthogonality. This situation arrived by selecting $(m-r)$ additional ortho normal vectors to form the matrix $E$. These additional vectors do not affect the final solution because the variances associated with these directions are zero. We select the matrix $P$ to be a matrix where each row $p i$ is an eigenvector of $X X T$. By this selection, we can say $P \equiv E T$. Substituting into Equation, we find $A=P T D P$. With this relation $(P-1=P T)$ we can finish evaluating $C Y$.

$$
\begin{aligned}
& C Y \equiv \frac{1}{n-1} \times P A P^{\mathrm{T}} \\
& C Y \equiv \frac{1}{n-1} \times \mathrm{P}\left(\mathrm{P}^{\mathrm{T}} \mathrm{DP}\right) \mathrm{P}^{\mathrm{T}} \\
& C Y \equiv \frac{1}{n-1} \times\left(P P^{\mathrm{T}}\right) \mathrm{D}\left(\mathrm{PP}^{\mathrm{T}}\right) \\
& C Y \equiv \frac{1}{n-1} \times\left(\mathrm{PP}^{-1}\right) \mathrm{D}\left(\mathrm{PP}^{-1}\right)
\end{aligned}
$$

$C Y \equiv \frac{1}{n-1} \times \mathrm{D}(1)$

It is evident that the choice of $P$ diagonalizes $C Y$. This was the goal for PCA.

\subsection{Eigen faces}

Eigen face method for human face recognition is remarkably clean and simple. The basic concept behind the Eigen face method is information reduction. When one evaluates even a small image, there is an incredible amount of information present. From all the possible things that could be represented in a given image, pictures of things that look like faces clearly represent a small portion of this image space. We seek a method to break down pictures that will be better equipped to represent face images rather than images in general. To do this, one should generate 'base-faces' and then represent any image being analyzed by the system as a linear combination[10-12] of these base faces. Once the base faces have been chosen we have essentially reduced the complexity of the problem from one of image analysis to a standard classification problem. Each face that we wish to classify can be projected into face-space and then analyzed as a vector. A k-nearest-neighbor approach, a neural network or even a simply Euclidian distance measure can be used for classification. The technique discussed in [13-14] can be broken down into the following components:

a. Generate the eigenfaces.

b. Project training data into face-space to be used with a predetermined classification method.

c. Evaluate a projected test element by projecting it into face space and comparing to training data.

\subsection{Generation of Eigen faces}

Before doingany work to generate the Eigen faces, sample faces are needed. These images will be used as examples of what an image in face-space looks like. These images do not need to be images of the people, the system will later be used to identify (though it can help); however the image should represent variations one would expect to see in the data on which the system is expected to be used, such as head tilt/angle, a variety of shading conditions, etc., Ideally these images should contain pictures of faces at close to the same scale, although this can be accomplished through preprocessing if necessary. It is required that all images being used in the system, both sample and test images, should be of same size. The resulting Eigen faces will also be of this same size once they have been calculated.

It is assumed that all images being dealt with are grayscale images, with pixel intensity values ranging from 0 to 255 . Suppose, there are $K$ images in our data set. Each sample image will be referred to as $X i$ where nindicates that we are dealing with $i$ th sample image $(1<=i<=\mathrm{K})$. Each $X i$ is a column vector. Generally images are thought of as pixels, each having $(x, y)$ coordinates with $(0,0)$ being at the upper left corner (or one could think of an image as a matrix with $y$ rows and $x$ columns). Converting this to a column form is a matter of convenience, it can be done in either column or row major form, so long as it is done consistently for all sample images, it will not affect the outcome. The size of the resulting $X i$ column vector will depend on the size of the sample images. If the sample images are $x$ pixels across and $y$ pixels tall, the column vector will be of size $(x * y) \times 1$. These original image sizes must be remembered if one wishes to view the resulting Eigen faces, or projections of test images 
into face-space. This is to allow a normal image to beconstructed from a column vector of image pixels. Let

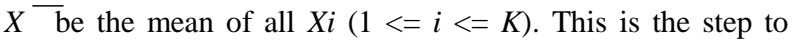
calculate an average face of the database if one were to reinterpret the vector as a normal image.

The next step is to calculate difference faces $U i$ such that $U i=$ $X i-X$ - (where $X$ - is mean) and form a matrix $U$, such that $U=$ $\left[\begin{array}{lll}U 1 & U 2 \ldots U K\end{array}\right]$. Our goal now is to generate the Eigen faces which is done by calculating the eigenvectors of the covariance matrix $U U T$. This cannot be done directly as the size of $U U T$ is $\left(x^{*} y\right)^{*}(x * y)$ which is very large. Clearly, doing these calculations on a resulting matrix of this size is going to be taxing on all but the most specialized, advance hardware. To avoid this problem, a trick from linear algebra is applied [17-18]. The eigenvectors of the UUT matrix can actually be found by considering linear combinations of the eigenvectors of the $U T U$ matrix. This is extremely useful when one realizes that the size of the $U T U$ matrix is $K \times K$. where in real world situations $K \ll\left(x^{*} y\right)$. The eigenvectors Wjof this matrix can be readily found through the following formula:

$W_{j}=\frac{\sum_{l=1}^{k} U_{l} E_{l j}}{\sqrt{\lambda_{j}}}$

Where Eljis the $l$ th value of thejth eigenvector of $U T U$ and $\lambda j$ is the corresponding Eigen value of Wjand $E j$. The linear algebra part of this trick is given below: Let the eigenvectors of $U T U$ will be

$E j(1<=j<=K)$ and the corresponding Eigen values be $\lambda j$. Hence, we can write:

$\mathrm{U}^{\mathrm{T}} \mathrm{U} \mathrm{E}_{\mathrm{J}}=\lambda_{j} E_{j}$

Multiplying both the sides by $U$ :

$\mathrm{U} \times \mathrm{U}^{\mathrm{T}} \mathrm{UE}_{\mathrm{j}}=\lambda_{\mathrm{j}} \mathrm{UE}_{\mathrm{j}}$

Thus, $\mathrm{W} j=U E j \mathrm{is}$ the $j$ theigenvector of UUT with corresponding Eigen value $\lambda j$. The fact that the Eigen values for the $U U T$ and $U T U$ are the same (though if we were going to calculate all Eigen values of the UUT matrix, we could get more values, the eigenvectors of $U T U$ only represent the most important subset of the Eigen values of the $U U T$ matrix).

\section{EXPERIMENTAL RESULTS}

\subsection{Registration}

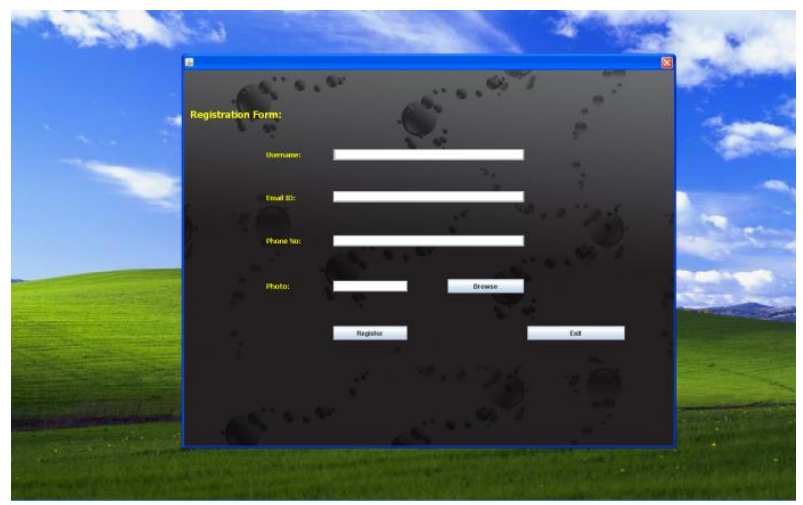

Fig: 2. Registration screen

\subsection{Login}

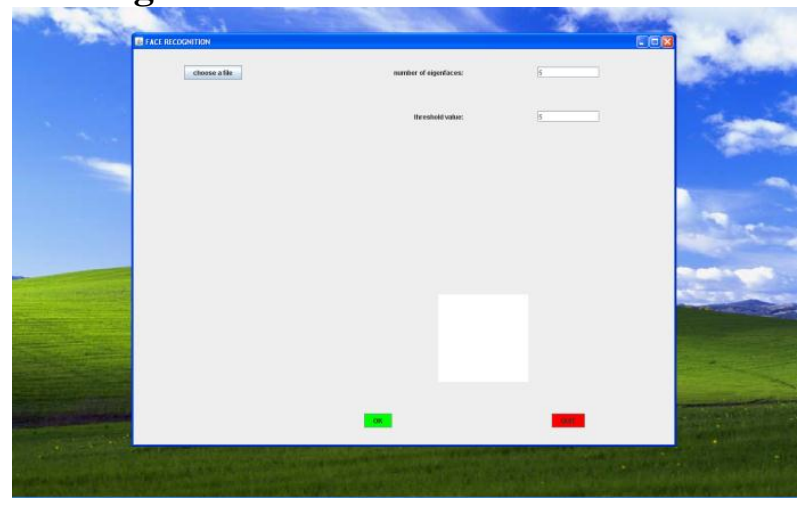

Fig: 3. Entering into the system with face recognization

\subsection{Image Validation}

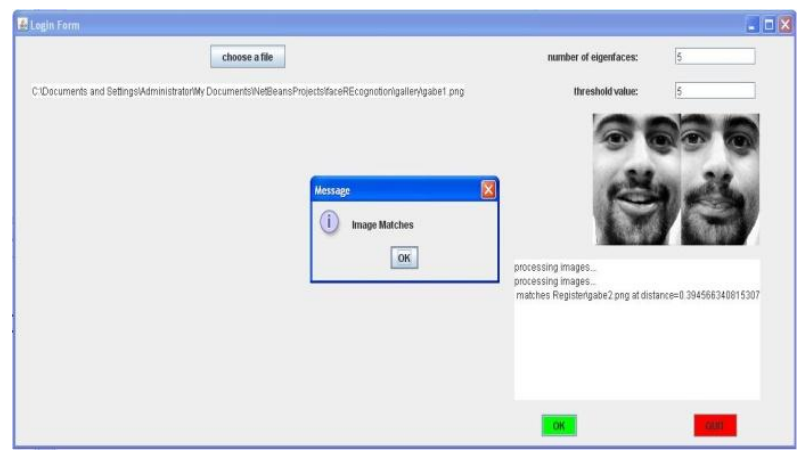

Fig: 4. Face matching with different expressions

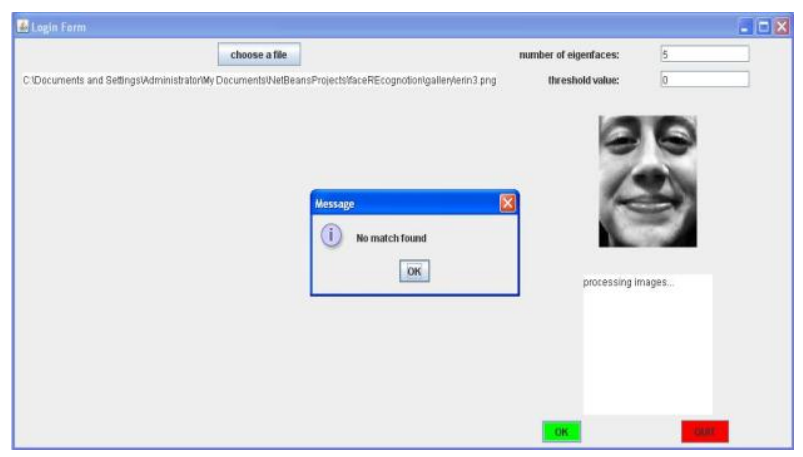

Fig: 5. Invalid match

\subsection{Details}

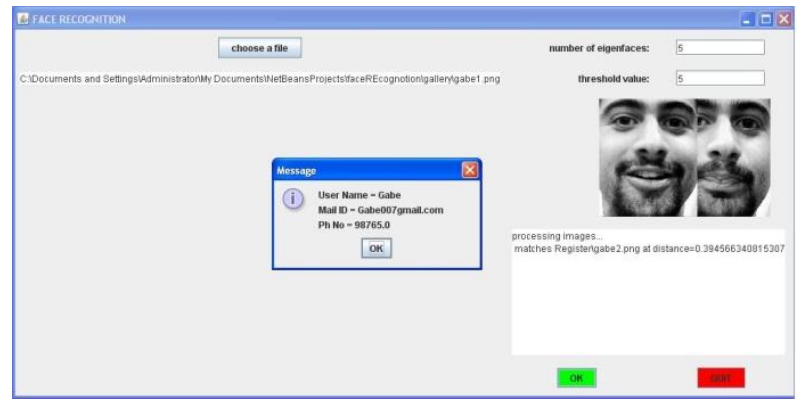

Fig: 6. User details tracking by PCASA matching 


\section{CONCLUSIONS}

In this paper we proposed a new face recognition system that uses eigenvectors and faces.This is calculated by using Principle Component Analysis Signcryption Algorithm (PCASA) which is more secure than existing systems.An analysis between PCA and PCASA is in process. Aging also one of the complexity of the image matching system.

\section{ACKNOWLEDGMENTS}

Our sincere thanks to all who have helped towards the development of this paper.

\section{REFERENCES}

[1] Wendy S. Yambor Bruce A. Draper J. Ross Beveridge, "Analyzing PCAbased Face Recognition Algorithms: Eigenvector Selection and DistanceMeasures", July 1, $2000 . \quad$ Available at:http://www.cs.colostate.edu/ vision/publications/eemc vcsu2000.pdf

[2] Peter Belhumeur, J. Hespanha, David Kriegman, "Eigenfaces vs.fisherfaces: Recognition using class specific linear projection", IEEETransactions on Pattern Analysis and Machine Intelligence, 19(7):771 -720, 1997.

[3] L. Breiman. Bagging predictors. Technical Report Technical ReportNumber 421, Dept. of Statistics, University of California, Berkeley,1994.

[4] D. Swets and J. Weng, "Hierarchical discriminant analysis for imageretrieval", IEEE Transactions on Pattern Analysis and MachineIntelligence, 21(5):386401, 1999.

[5] Wendy S. Yambor, "Analysis of PCA Based and Fisher Discriminant-Based Image Recognition Algorithms", M.S. Thesis, July 2000(Technical Report CS-00-103, Computer Science).

[6] Kyungnam Kim, "Face Recognition using Principle ComponentAnalysis", International Conference on Computer Vision and PatternRecognition, pp. 586-591, 1996. [7]http://scien.stanford.edu/class/ee368/projects2001/dropbox/ project16/

[8] http://www.irc.atr.jp/\%7Emlyons/pub_pdf/fg98-1.pdf

[9] http://www.kasrl.org/jaffe.html

[10] James R. Parker, J R Parker, "Algorithms for Image Processing andComputer Vision", John Wiley \& Sons, 1996.

[11] Sankar K. Pal, AshishGhosh, Malay K. Kundu, "Soft Computing forImage Processing", Studies in Fuzziness and Soft Computing, Vol. 42,2000.

[12] Rafael C. Gonzalez, Richard E. Woods, "Digital Image Processing",Pearson Publications, 2000.

[13] Image Processing Handbook by John C. Russ

[14] Handbook of Pattern Recognition and Image Processing by K.S. Fu andT.Y. Young

[15] Li Ma , Tieniu Tan , Yunhong Wang , Dexin Zhang “ PersonalIdentification Based on Iris Texture Analysis", IEEE Transactions onPattern Analysis and Machine Intelligence, Vol. 25 No. 12, December2003.

[16] John Carter, Mark Nixon, "An Integrated Biometric Database",available at:ieeexplore.ieee.org/iel3/1853/4826/00190224.pdf.

[17] Arun Rose, Anil Jain and SharatPankanti, "A Prototype HandGeometry Based Verification System", 2nd International Conference onAudio and Video Based Person Authentication, Washington D. C., pp.166-171, 1999.

[18] Boreki, Guilherm, Zimmer, Alessandro, "Hand Geometry FeatureExtraction through Curvature Profile Analysis", XVIII BrazilianSymposium on Computer Graphics and Image Processing, SIBGRAPI,Brazil, 2005. 